\title{
Optimal Packet Scheduling in a Broadcast Channel with an Energy Harvesting Transmitter
}

\author{
Jing Yang Omur Ozel Sennur Ulukus \\ Department of Electrical and Computer Engineering \\ University of Maryland, College Park, MD 20742 \\ yangjing@umd.edu_omur@umd.edu_ulukus@umd.edu
}

\begin{abstract}
In this paper, we investigate the transmission completion time minimization problem in a two-user additive white Gaussian noise (AWGN) broadcast channel, where the transmitter is able to harvest energy from the nature. The harvested energy is modeled to arrive at the transmitters randomly. In this paper, under a deterministic system setting, we assume that the energy harvesting times and harvested energy amounts are known before the transmission starts. The transmitter has a fixed number of packets to be delivered to each receiver. Our goal is to minimize the time by which all of the packets for both users are delivered to their respective destinations. To this end, we optimize the transmit powers and transmission rates intended for both users. We first analyze the structural properties of the optimal transmission policy. We prove that the optimal total transmit power has the same structure as the optimal single-user transmit power [1], [2]. We also prove that there exists a cut-off power level for the stronger user. If the optimal total transmit power is lower than this level, all transmit power is allocated to the stronger user, and when the optimal total transmit power is larger than this level, all transmit power above this level is allocated to the weaker user. Based on these structural properties of the optimal policy, we propose an algorithm that yields the globally optimal off-line scheduling policy.
\end{abstract}

\section{INTRODUCTION}

We consider a wireless communication network where users are able to harvest energy from the nature. Such energy harvesting capabilities make sustainable and environmentally friendly deployment of wireless communication networks possible. While energy-efficient scheduling policies have been well-investigated in traditional battery powered (unrechargeable) systems [3]-[6], energy-efficient scheduling in energy harvesting networks with nodes that have rechargeable batteries has only recently been considered [1], [2]. References [1], [2] consider a single-user communication system with an energy harvesting transmitter, and develop a packet scheduling scheme that minimizes the time by which all of the packets are delivered to the receiver.

In this paper, we consider a multi-user extension of the work in [1], [2]. In particular, we consider a wireless broadcast channel with an energy harvesting transmitter. As shown in Fig. 1, we consider a broadcast channel with one transmitter and two receivers, where the transmitter node has three queues. The data queues store the data arrivals intended for the individual receivers, while the energy queue stores the

This work was supported by NSF Grants CCF 04-47613, CCF 05-14846, CNS 07-16311, CCF 07-29127, CNS 09-64632. energy harvested from the environment. Our objective is to adaptively change the transmission rates that go to both users according to the instantaneous data and energy queue sizes, such that the transmission completion time is minimized.

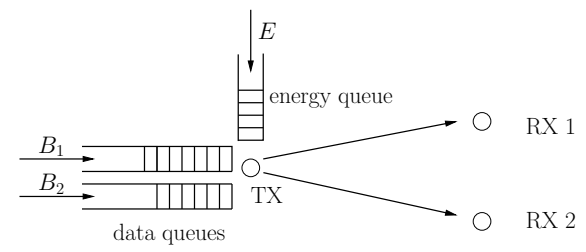

Fig. 1. An energy harvesting two-user broadcast channel.

In this paper, we focus on finding the optimum off-line schedule, by assuming that the energy arrival profile at the transmitter is known ahead of time in an off-line manner. We assume that there are a total of $B_{1}$ bits that need to be delivered to receiver 1 and $B_{2}$ bits that need to be delivered to receiver 2, at the transmitter. As shown in Fig 2, energy arrives (is harvested) at points in time marked with $\circ$; in particular, $E_{k}$ denotes the amount of energy harvested at time $s_{k}$. Our goal is to develop a method of transmission to minimize the time, $T$, by which all of the data packets are delivered to their respective receivers.

The optimal packet scheduling problem in a single-user energy harvesting communication system is investigated in [1], [2]. In [1], [2], we prove that the optimal scheduling policy has a "majorization" structure, in that, the transmit power is kept constant between energy harvests, the sequence of transmit powers increases monotonically, and only changes at some of the energy harvesting instances; when the transmit power changes, the energy constraint is tight, i.e., the total consumed energy equals the total harvested energy. In [1], [2], we develop an algorithm to obtain the optimal off-line scheduling policy based on these properties. Reference [7] extends [1], [2] to the case where rechargeable batteries have finite sizes. We extend [1], [2] in [8] for a fading channel and develop online algorithms.

References [7], [8] investigate two related problems. The first problem is to maximize the throughput (number of bits transmitted) with a given deadline constraint, and the second problem is to minimize the transmission completion time with a given number of bits to transmit. These two problems are "dual" to each other in the sense that, with a given energy arrival profile, if the maximum number of bits that can be sent 


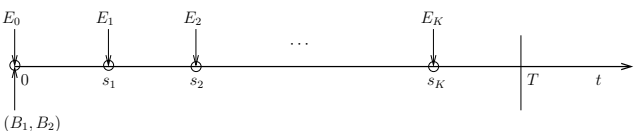

Fig. 2. System model. $\left(B_{1}, B_{2}\right)$ bits to be transmitted to users are available at the transmitter at the beginning. Energies arrive (are harvested) at points denoted by $0 . T$ denotes the transmission completion time by which all of the bits are delivered to their respective destinations.

by a deadline is $B^{*}$ in the first problem, then the minimum time to transmit $B^{*}$ bits in the second problem must be the deadline in the first problem, and the optimal transmission policies for these two problems must be identical. In this paper, we will follow this "dual problems" approach. We will first attack and solve the first problem to determine the structural properties of the optimal solution. We will then utilize these structural properties to develop an iterative algorithm for the second problem. Our iterative approach has the goal of reducing the two-user broadcast problem into a single-user problem as much as possible, and utilizing the single-user solution in [1], [2]. The second problem is also studied independently in [9] where an iterative solution is proposed that considers local sub-problems with only two energy arrivals at a time.

We first analyze the structural properties of the optimal policy for the first problem where our goal is to maximize the number of bits delivered to both users under a given deadline constraint. To that end, we first determine the maximum departure region with a given deadline constraint $T$. The maximum departure region is defined as the set of all $\left(B_{1}, B_{2}\right)$ that can be transmitted to users reliably with a given deadline. In order to do that, we consider the problem of maximizing $\mu_{1} B_{1}+\mu_{2} B_{2}$ under the energy causality constraints for the transmitter, for all $\mu_{1}, \mu_{2} \geq 0$. Varying $\mu_{1}, \mu_{2}$ traces the boundary of the maximum departure region. We prove that the optimal total transmit power policy is independent of the values of $\mu_{1}, \mu_{2}$, and it has the same "majorization" structure as the single-user non-fading solution. As for the way of splitting the total transmit power between the two users, we prove that there exists a cut-off power level for the stronger user, i.e., only the power above this cut-off power level is allocated to the weaker user.

We then consider the second problem, where our goal is to minimize the time, $T$, by which a given $\left(B_{1}, B_{2}\right)$ number of bits are delivered to their intended receivers. As discussed, since the second problem is "dual" to the first problem, the optimal transmission policy in this problem has the same structural properties as in the first problem. Therefore, in the second problem as well, there exists a cut-off power level. The problem then becomes that of finding an optimal cut-off power such that the transmission times for both users become identical and minimized. With these optimal structural properties, we develop an iterative algorithm that finds the optimal schedule efficiently. In particular, we first use the fact that the optimum transmit power has the same structural properties as the single-user problem, to obtain the first optimal total power, $P_{1}$. Then, given the fact that there exists a cut-off power level, $P_{c}$, for the first user, the optimal transmit strategy depends on whether $P_{1}$ is smaller or larger than $P_{c}$, which, at this point, is unknown. Therefore, we have two cases to consider. If $P_{c}$ is smaller than $P_{1}$, then the stronger user will always have a constant, $P_{c}$, portion of the total transmit power. This reduces the problem to a singleuser problem for the second user, together with a fixed-point equation in a single variable $\left(P_{c}\right)$ to be solved to ensure that the transmissions to both users end at the same time. On the other hand, if $P_{c}$ is larger than $P_{1}$, this means that all of $P_{1}$ must be spent to transmit to the first user. In this case, the number of bits delivered to the first user in this time duration can be subtracted from the total number of bits to be delivered to the first user, and the problem can be started anew with the updated number of bits $\left(B_{1}^{\prime}, B_{2}\right)$ after the first epoch. Therefore, in both cases, the broadcast channel problem is essentially reduced to single-user problems, and the approach in [1], [2] is utilized recursively to solve the overall problem.

\section{System Model AND PROBlem Formulation}

The system model is as shown in Figs. 1 and 2. The transmitter has an energy queue and two data queues (Fig. 1). The physical layer is modeled as an AWGN broadcast channel, where the received signals at the two receivers are

$$
\begin{aligned}
& Y_{1}=X+Z_{1} \\
& Y_{2}=X+Z_{2}
\end{aligned}
$$

where $X$ is the transmit signal, and $Z_{1}$ is a Gaussian noise with zero-mean and unit-variance, and $Z_{2}$ is a Gaussian noise with zero-mean and variance $\sigma^{2}$, where $\sigma^{2}>1$. Therefore, the second user is the degraded (weaker) user in our broadcast channel. Assuming that the transmitter transmits with power $P$, the capacity region for this two-user AWGN broadcast channel is [10]

$$
\begin{aligned}
& r_{1} \leq \frac{1}{2} \log _{2}(1+\alpha P) \\
& r_{2} \leq \frac{1}{2} \log _{2}\left(1+\frac{(1-\alpha) P}{\alpha P+\sigma^{2}}\right)
\end{aligned}
$$

where $\alpha$ is the fraction of power spent for the message transmitted to the first user.

Working on the boundary of the capacity region, we have

$$
\begin{aligned}
P & =2^{2\left(r_{1}+r_{2}\right)}+\left(\sigma^{2}-1\right) 2^{2 r_{2}}-\sigma^{2} \\
& \triangleq g\left(r_{1}, r_{2}\right)
\end{aligned}
$$

As shown in Fig. 1, the transmitter has $B_{1}$ bits to transmit to the first user, and $B_{2}$ bits to transmit to the second user. Energy is harvested at times $s_{k}$ with amounts $E_{k}$. Our goal is to develop a transmission policy that minimizes the time, $T$, by which all of the bits are delivered to their intended receivers. The transmitter adapts its transmit power and the portions of the total transmit power used to transmit signals to the two users according to the available energy level and the remaining number of bits. The energy consumed must satisfy the causality constraints, i.e., at any given time, the total amount of energy consumed up to time $t$ must be less 
than or equal to the total amount of energy harvested up to time $t$.

Before we proceed to give a formal definition of the optimization problem and propose the solution, we start with the "dual" problem of this transmission completion time minimization problem, i.e, instead of trying to find the minimal $T$, we aim to identify the maximum number of bits the transmitter can deliver to both users by any fixed time $T$. As we will observe in the next section, solving the "dual" problem enables us to identify the optimal structural properties for both problems, and these properties eventually help us reduce the original problem into simple scenarios, which can be solved efficiently.

\section{Characterizing $\mathcal{D}(T)$ : Largest $\left(B_{1}, B_{2}\right)$ Region FOR A GIVEN $T$}

In this section, our goal is to characterize the maximum departure region for a given deadline $T$. We define it in the following way.

Definition 1 For any fixed transmission duration $T$, the maximum departure region, denoted as $\mathcal{D}(T)$, is the union of $\left(B_{1}, B_{2}\right)$ under any feasible rate allocation policy over $d u$ ration $[0, T)$, i.e., $\mathcal{D}(T)=\bigcup_{r_{1}(t), r_{2}(t)}\left(B_{1}, B_{2}\right)\left(r_{1}(t), r_{2}(t)\right)$, subject to the energy constraint $\int_{0}^{t} g\left(r_{1}, r_{2}\right)(\tau) d \tau \leq$ $\sum_{i: s_{i}<t} E_{i}$.

We call any policy optimal if it achieves the boundary of $\mathcal{D}(T)$. In the single-user scenario in [1], we first examined the structural properties of the optimal policy. Based on these properties, we developed an algorithm to find the optimal scheduling policy. In this broadcast scenario, we will first analyze the structural properties of the optimal policy, and then obtain the optimal solution based on these structural properties. The following lemma which was proved for a single-user problem in [1], [2] was also proved for the broadcast problem in [9].

Lemma 1 Under the optimal policy, the transmission rate remains constant between energy harvests, i.e., the rate only potentially changes at an energy harvesting epoch.

Due to space limitations, the proof of this, and all upcoming lemmas, will be omitted here, and can be found in [11].

Therefore, in the following, we only consider policies where the rates are constant between any two consecutive energy arrivals. We denote the rates that go to both users as $\left(r_{1 n}, r_{2 n}\right)$ over the duration $\left[s_{n-1}, s_{n}\right)$.

Lemma $2 \mathcal{D}(T)$ is a convex region.

In order to simplify the notation, in this section, for any given $T$, we assume that there are $N-1$ energy arrival epochs (excluding $t=0)$ over $(0, T)$. We denote the last energy arrival epoch before $T$ as $s_{N-1}$, and $s_{N}=T$, with $l_{n}=$ $s_{n}-s_{n-1}$ for $n \leq N$, as shown in Fig. 3 .

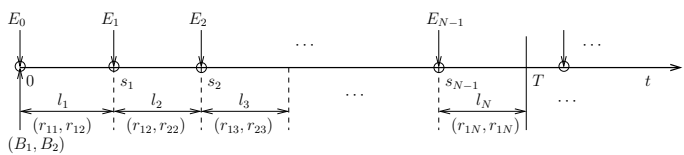

Fig. 3. Rates $\left(r_{1 n}, r_{2 n}\right)$ and corresponding durations $l_{n}$ with a given deadline $T$

Since $\mathcal{D}(T)$ is a strictly convex region, its boundary can be characterized by solving the following optimization problem for all $\mu_{1}, \mu_{2} \geq 0$,

$$
\begin{aligned}
\max _{\mathbf{r}_{1}, \mathbf{r}_{2}} & \mu_{1} \sum_{n=1}^{N} r_{1 n} l_{n}+\mu_{2} \sum_{n=1}^{N} r_{2 n} l_{n} \\
\text { s.t. } & \sum_{n=1}^{j} g\left(r_{1 n}, r_{2 n}\right) l_{n} \leq \sum_{n=0}^{j-1} E_{n}, \quad 0<j \leq N
\end{aligned}
$$

where $\mathbf{r}_{1}$ and $\mathbf{r}_{2}$ denote the rate sequences $r_{1 n}$ and $r_{2 n}$ for users 1 and 2, respectively. The problem in (7) is a convex optimization problem with convex cost function and linear constraints, therefore, the unique global solution should satisfy the extended KKT conditions. Based on these KKT optimality conditions, we can prove an important property of the optimal policy.

Lemma 3 The optimal total transmit power of the transmitter is independent of the value of $\mu_{1}, \mu_{2}$, and it is the same as the single-user optimal transmit power. Specifically,

$$
\begin{aligned}
& i_{n}=\arg \min _{i_{n-1}<i \leq N}\left\{\frac{\sum_{j=i_{n-1}}^{i-1} E_{j}}{s_{i}-s_{i_{n-1}}}\right\} \\
& P_{n}=\frac{\sum_{j=i_{n-1}}^{i_{n}-1} E_{j}}{s_{i_{n}}-s_{i_{n-1}}}
\end{aligned}
$$

i.e., at $t=s_{i_{n}}, P_{n}$ switches to $P_{n+1}$.

Since the power can be obtained directly irrespective of the values of $\mu_{1}, \mu_{2}$, the optimization problem in (7) is separable over each duration $\left[s_{n-1}, s_{n}\right)$. Specifically, for $0<n \leq N$, the local optimization becomes

$$
\begin{aligned}
\max _{r_{1 n}, r_{2 n}} & \mu_{1} r_{1 n}+\mu_{2} r_{2 n} \\
\text { s.t. } & g\left(r_{1 n}, r_{2 n}\right) \leq P_{n}
\end{aligned}
$$

Let us define a constant power level as

$$
P_{c}=\left(\frac{\mu_{1}\left(\sigma^{2}-1\right)}{\mu_{2}-\mu_{1}}-1\right)^{+}
$$

Based on the solution of the local optimization problem (10), we establish another important property of the optimal policy as follows.

Lemma 4 For fixed $\mu_{1}, \mu_{2}$, under the optimal power policy, there exists a constant cut-off power level, $P_{c}$, for the first user. If the total power level is below this cut-off power level, then, all the power is allocated to the first user; if the power level is higher than this level, then, all the power above this cut-off level is allocated to the second user. 
Combining Lemma 3 and Lemma 4, we illustrate the structure of the optimal policy in Fig. 4.

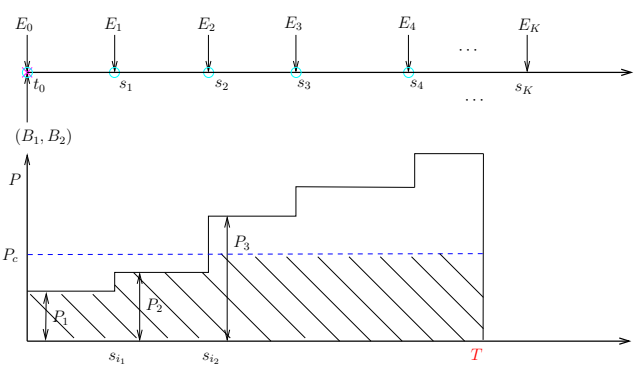

Fig. 4. Optimally splitting total power between the signals that go to the two users.

Based on Lemma 3, we observe that for fixed $T, \mu_{1}$ and $\mu_{2}$, the optimal power allocation is unique, i.e., does not depend on $\mu_{1}$ and $\mu_{2}$. However, the way the total power is split between the two users depends on $\mu_{1}, \mu_{2}$. In fact, the cut-off power level $P_{c}$ varies depending on the value of $\mu_{2} / \mu_{1}$. Therefore, for different values of $\mu_{2} / \mu_{1}$, the optimal policy achieves different boundary points on the maximum departure region, and varying the value of $\mu_{2} / \mu_{1}$ traces the boundary of this region.

In this section, we characterized the maximum departure region for any given time $T$. We proved that the optimal total transmit power is the same as in the single-user case, and there exists a cut-off power for splitting the total transmit power to both users. In the next section, we will use these structural properties to solve the original transmission completion minimization problem.

\section{Minimizing the Transmission Completion Time $T$ FOR A GIVEN $\left(B_{1}, B_{2}\right)$}

In this section, our goal is to minimize the transmission completion time of both users for a given $\left(B_{1}, B_{2}\right)$. The optimization problem can be formulated as

$$
\begin{array}{ll}
\min _{\mathbf{r}_{1}, \mathbf{r}_{2}} & T \\
\text { s.t. } & \sum_{n=1}^{j} g\left(r_{1 n}, r_{2 n}\right) l_{n} \leq \sum_{n=1}^{j-1} E_{n}, \quad 0<j \leq N(T) \\
& \sum_{n=1}^{N(T)} r_{1 n} l_{n}=B_{1}, \quad \sum_{n=1}^{N(T)} r_{1 n} l_{n}=B_{2}
\end{array}
$$

where $N(T)-1$ is the number of energy arrival epochs (excluding $t=0$ ) over $(0, T)$, and $l_{N(T)}=T-s_{N(T)-1}$. Since $N(T)$ depends on $T$, the optimization problem in (12) is not a convex optimization problem in general. Therefore, we cannot solve it using standard convex optimization tools.

We first note that this is exactly the "dual" problem of maximizing the departure region for fixed $T$. They are "dual" in the sense that, if the minimal transmission completion time for $\left(B_{1}, B_{2}\right)$ is $T$, then $\left(B_{1}, B_{2}\right)$ must lie on the boundary of $\mathcal{D}(T)$, and the transmission policy should be exactly the same for some $\left(\mu_{1}, \mu_{2}\right)$. This is based on the fact that $\mathcal{D}(T) \subset$ $\mathcal{D}\left(T^{\prime}\right)$ for any $T<T^{\prime}$. Assume $\left(B_{1}, B_{2}\right)$ does not lie on the boundary of $\mathcal{D}(T)$. Then, either $\left(B_{1}, B_{2}\right)$ cannot be achieved by $T$ or $\left(B_{1}, B_{2}\right)$ is strictly inside $\mathcal{D}(T)$ and hence $\left(B_{1}, B_{2}\right)$ can be achieved by $T^{\prime}<T$. Therefore, if $\left(B_{1}, B_{2}\right)$ does not lie on the boundary of $\mathcal{D}(T)$, then $T$ cannot be the minimum transmission completion time.

Lemma 5 When $B_{1}, B_{2} \neq 0$, under the optimal policy, the transmissions to both users must be finished at the same time.

For fixed $\left(B_{1}, B_{2}\right)$, let us denote the transmission completion time for the first and second user, by $T_{1}, T_{2}$, respectively. We note that $T_{1}$ and $T_{2}$ depend on the selection of the cut-off power level, $P_{c}$. In particular, $T_{1}$ is monotonically decreasing in $P_{c}$, and $T_{2}$ is monotonically increasing in $P_{c}$. Based on Lemma 5, the problem of optimal selection of $P_{c}$, can be viewed as solving a fixed point equation. In particular, $P_{c}$ must be chosen such that, the resulting $T_{1}$ equals $T_{2}$. Therefore, we propose the following algorithm to solve the transmission completion time, $T$, minimization problem. Our basic idea is to try to identify the cut-off power level $P_{c}$ in an efficient way.

Since the power allocation is similar to the single-user case (c.f. Lemma 3), our approach to find $T$ will be similar to the method in [1], [2]. First, we aim to identify $P_{1}$, the first total transmit power starting from $t=0$ in the system. This is exactly the same as identification of $P_{1}$ in the corresponding single-user problem. For this, as in [1], [2], we treat the energy arrivals as if they have arrived at time $t=0$, and obtain a lower bound for the transmission completion time as in [1], [2]. In order to do that, first, we compute the amount of energy required to finish $\left(B_{1}, B_{2}\right)$ by $s_{1}$. This is equal to $g\left(\frac{B_{1}}{s_{1}}, \frac{B_{2}}{s_{1}}\right) s_{1}$, denoted as $A_{1}$. Then, we compare $A_{1}$ with $E_{0}$. If $E_{0}$ is greater than $A_{1}$, this implies that the transmitter can finish the transmission before $s_{1}$ with $E_{0}$, and future energy arrivals are not needed. In this case, the minimum transmission completion time is the solution of

$$
g\left(\frac{B_{1}}{T}, \frac{B_{2}}{T}\right) T=E_{0}
$$

If $A_{1}$ is greater than $E_{0}$, this implies that the final transmission completion time is greater than $s_{1}$, and some of the future energy arrivals must be utilized to complete the transmission. We calculate the amount of energy required to finish $\left(B_{1}, B_{2}\right)$ by $s_{2}, s_{3}, \ldots$, and denote them as $A_{2}, A_{3}$, $\ldots$, and compare these with $E_{0}+E_{1}, \sum_{j=0}^{2} E_{j}, \sum_{j=0}^{3} E_{j}$, $\ldots$, until the first $A_{i}$ that becomes smaller than $\sum_{j=0}^{i-1} E_{j}$. We denote the corresponding time index as $\tilde{i}_{1}$. Then, we assume that we can use $\sum_{i=0}^{\tilde{i}_{1}-1} E_{i}$ to transmit $\left(B_{1}, B_{2}\right)$ at a constant rate. And, the corresponding transmission completion time is the solution of the following equation

$$
g\left(\frac{B_{1}}{T}, \frac{B_{2}}{T}\right) T=\sum_{i=0}^{\tilde{i}_{1}-1} E_{i}
$$

We denote the solution to this equation as $\tilde{T}$, and the corresponding power as $\tilde{P}_{1}$. From our analysis, we know that the solution to this equation is the minimum possible transmission completion time we can achieve. Then, we check 
whether this constant power $\tilde{P}_{1}$ is feasible, when the actual energy arrival times are imposed. If it is feasible, it gives us the minimal transmission completion time; otherwise, we get $P_{1}$ by selecting the minimal slope according to (9). That is to say, we draw all of the lines from $t=0$ to the corner points of the energy arrival instances before $\widetilde{T}$, and choose the line with the smallest slope. We denote $s_{i_{1}}$ as the corresponding duration associated with $P_{1}$. This is shown in Fig. 5.

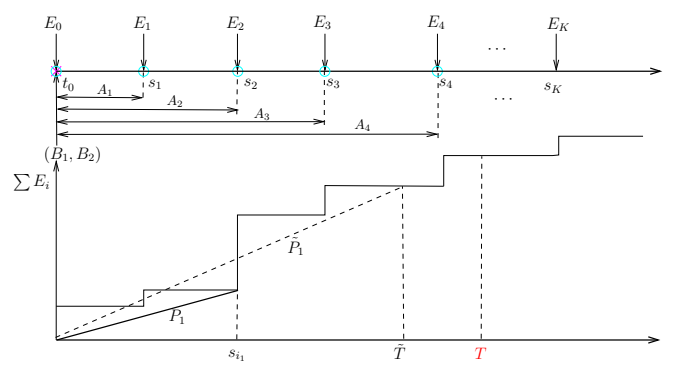

Fig. 5. Determining the optimal total power level of the first epoch.

Once $P_{1}$ is selected, we know that it is the optimal total transmit power in our broadcast channel problem. Next, we need to divide this total power between the signals transmitted to the two users. Based on Lemma 4, if the cutoff power level $P_{c}$ is higher than $P_{1}$, then, the transmitter spends all $P_{1}$ for the stronger user; otherwise, the first user finishes its transmission with a constant power $P_{c}$.

We will first determine whether $P_{c}$ lies in $\left[0, P_{1}\right]$ or it is higher than $P_{1}$. Assume $P_{c}=P_{1}$. The transmission completion time for the first user is

$$
T_{1}=\frac{B_{1}}{f\left(P_{1}\right)}
$$

where $f(P)=\frac{1}{2} \log (1+P)$. Once $P_{c}$ is fixed, we can obtain the minimum transmission completion time for the second user, $T_{2}$, by subtracting the energy consumed for the first user, and treating $P_{1}$ as a constant interference for the second user. If $T_{1}=T_{2}$, this value is the minimum transmission completion time. To find the minimum transmission completion time recursively, we calculate the maximum number of bits departed from the second user by the deadline $T_{1}$ and denote it as $D_{2}\left(T_{1}, P_{c}\right)$. We have

$$
D_{2}\left(T_{1}, P_{c}\right)=\sum_{i=1}^{N\left(T_{1}\right)} \frac{1}{2} \log \left(1+\frac{P_{n}^{*}-P_{c}}{P_{c}+\sigma^{2}}\right)\left(s_{i_{n}}-s_{i_{n-1}}\right)
$$

where $P_{1}^{*}, P_{2}^{*}, \ldots, P_{N\left(T_{1}\right)}^{*}$ is the optimal power allocation by the fixed deadline $T_{1}$. Note that $P_{1}^{*}=P_{1}$.

If $D_{2}\left(T_{1}, P_{c}\right)$ is smaller than $B_{2}$, it implies that $T_{1}<T_{2}$, and we need to decrease the rate for the first user to make $T_{1}$ and $T_{2}$ equal. This also implies from Lemma 4 that $P_{c}<$ $P_{1}$. In particular, $P_{c}$ is the unique solution of the following equation.

$$
B_{2}=D_{2}\left(\frac{B_{1}}{f\left(P_{c}\right)}, P_{c}\right)
$$

It can be shown that $D_{2}\left(\frac{B_{1}}{f\left(P_{c}\right)}, P_{c}\right)$ is a continuous, strictly monotonically decreasing function of $P_{c}$, hence the solution for $P_{c}$ in (16) is unique. Since $T_{1}$ is a decreasing function of $P_{c}$ and $D_{2}\left(\frac{B_{1}}{f\left(P_{c}\right)}, P_{c}\right)$ is a decreasing function of $P_{c}$, we can use the bisection method on $P_{c}$ to solve (16). In this case, the minimum transmission completion time is $T=\frac{B_{1}}{f\left(P_{c}\right)}$.

If $D_{2}\left(T_{1}, P_{c}\right)$ is larger than $B_{2}$, that implies $T_{2}<T_{1}$, and we need to increase the power allocated for the first user to make $T_{1}=T_{2}$, i.e., $P_{c}>P_{1}$. Therefore, from Lemma 4, over the duration $\left[0, s_{i_{1}}\right)$, the optimal policy is to allocate the entire $P_{1}$ to the first user only. We allocate $P_{1}$ to the first user, calculate the number of bits departed for the first user, and remove them from $B_{1}$. This simply reduces the problem to that of transmitting $\left(B_{1}^{\prime}, B_{2}\right)$ bits starting at time $t=s_{i_{1}}$, where $B_{1}^{\prime}=B_{1}-f\left(P_{1}\right) s_{i_{1}}$. The process is illustrated in Fig. 6. Then, the minimum transmission completion time is

$$
T=s_{i_{K}}+\frac{B_{1}-\sum_{k=1}^{K} f\left(P_{k}\right)\left(s_{i_{k}}-s_{i_{k-1}}\right)}{f\left(P_{c}\right)}
$$

where $K$ is the number of recursions needed to get $P_{c}$.

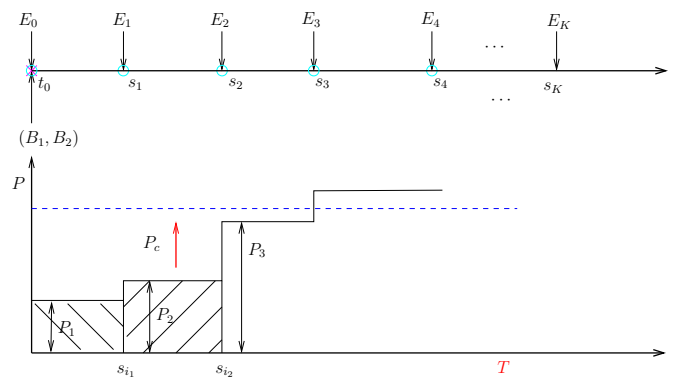

Fig. 6. Search for the cutoff power level $P_{c}$ iteratively.

In both scenarios, we reduce the problem into a simple form and obtain the global optimal policy.

\section{REFERENCES}

[1] J. Yang and S. Ulukus, "Transmission completion time minimization in an energy harvesting system," CISS, March 2010.

[2] _ - "Optimal packet scheduling in an energy harvesting communication system," IEEE Trans. Comm., submitted June 2010, also available at [arXiv: 1010.1295].

[3] E. Uysal-Biyikoglu, B. Prabhakar, and A. El Gamal, "Energy-efficient packet transmission over a wireless link," IEEE/ACM Trans. Networking, vol. 10, pp. 487-499, 2002.

[4] M. A. Zafer and E. Modiano, "A calculus approach to energy-efficient data transmission with quality of service constraints," IEEE/ACM Trans. Networking, vol. 17, pp. 898-911, June 2009.

[5] W. Chen, U. Mitra, and M. Neely, "Energy-efficient scheduling with individual delay constraints over a fading channel," WiOpt, Apr 2007.

[6] E. Uysal-Biyikoglu and A. El Gamal, "On adaptive transmission for energy efficiency in wireless data networks," IEEE Trans. Inform. Theory, vol. 50, pp. 3081-3094, December 2004.

[7] K. Tutuncuoglu and A. Yener, "Optimum transmission policies for battery limited energy harvesting nodes," IEEE Trans. Wireless Comm., submitted, September 2010, also available at [arXiv:1010.6280].

[8] O. Ozel, K. Tutuncuoglu, J. Yang, S. Ulukus, and A. Yener, "Transmission with energy harvesting nodes in fading wireless channels: Optimal policies," IEEE JSAC, submitted, October 2010.

[9] M. A. Antepli, E. Uysal-Biyikoglu, and H. Erkal, "Optimal packet scheduling on an energy harvesting broadcast link," IEEE JSAC, submitted, October 2010, also available at [arXiv:1010.4876].

[10] T. M. Cover and J. A. Thomas, Elements of Information Theory. New York: John Wiley and Sons, Inc, 1991.

[11] J. Yang, O. Ozel, and S. Ulukus, "Broadcasting with an energy harvesting rechargeable transmitter," IEEE Trans. Wireless Comm., submitted, October 2010, also available at [arXiv:1010.2993]. 